\title{
Multilayered ferromagnetic nanostructures study: processing data from magneto-ellipsometry measurements
}

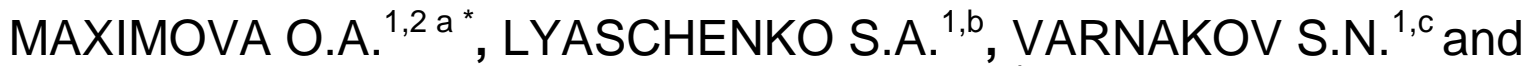 \\ OVCHINNIKOV S.G. ${ }^{1,2 d}$ \\ ${ }^{1}$ Kirensky Institute of Physics of SB RAS, 50/38 Akademgorodok, Krasnoyarsk 660036, Russia \\ ${ }^{2}$ Siberian Federal University, 79 Svobodny pr., Krasnoyarsk 660041, Russia \\ amaximo.a@mail.ru, Isa@iph.krasn.ru, ${ }^{\mathrm{c}} \mathrm{v}$ sn@@iph.krasn.ru, ${ }^{\mathrm{d}}$ sgo@iph.krasn.ru
}

Keywords: ellipsometry, ferromagnetic, thin layered films, transverse magneto-optical Kerr effect.

\begin{abstract}
Nowadays, the magneto-ellipsometry technique is considered as a promising tool for studying nanostructures. It leads to a great demand of both designing set-ups for conducting experiments and developing approaches to data processing. The later one is a problem in framework of in situ analysis as it would be useful to have an approach to data analysis which is reliable, quick and reasonably easy. This work continues our previous study of layered nanostructures by means of magneto-ellipsometry technique and logically generalizes the approach to magneto-ellipsometry data analysis for the multi-layered model use. As a result, the algorithm with detailed description of necessary formulae is presented.
\end{abstract}

\section{Introduction}

When creating new materials with specified functional properties, both the technology of their synthesis and the methods of non-destructive high-precision and high-speed control of the physical properties of such materials play an important role. In particular, when creating modern microwave devices, nanoelectronics devices and spintronics, there are technological problems in setting and controlling the imperfection of the crystal structure of ferromagnetic materials. The imperfection of a nanostructured ferromagnet actually determines its electronic structure, affects the electronic conductivity and the spin polarization of conduction electrons, plays role in the formation of the domain structure, determines the diffusion and thermodynamic properties of the material.

The most accurate and reliable data on the properties of a ferromagnetic nanostructure can be obtained from measurements conducted directly in the process chamber, that is, in situ, excluding the influence of the atmosphere on the sample surface. Few experimental methods allow in situ realization, and spectroscopic methods of analysis in the optical and infrared ranges of electromagnetic waves of small amplitude can be named among the methods which practically do not effect on the structure. One of such methods is the magnetically modulated reflective spectral ellipsometry (magneto-ellipsometry). At the moment there is a problem of reproducible and nondestructive in situ optical, magneto-optical and magnetic measurements, which includes the complexity of both the technical implementation of the optical system and of interpreting in situ results of magneto-optical measurements.

The reasons for the development of non-destructive in situ methods of analyzing thin ferromagnetic films are: an increase in the reproducibility of their physical properties during synthesis in an ultrahigh vacuum; a decrease in the characteristic dimensions of the synthesized ferromagnetic structures due to the exclusion of external influence on the surface. The use of surface-sensitive optical and magneto-optical methods of analysis in this case makes it possible additionally to investigate and control the physico-chemical processes occurring at the vacuum-film and film-substrate interfaces, as well as the state of the interlayer interfaces under the condition of optical transparency of the entire structure on substrate, i.e. for thicknesses up to $100 \mathrm{~nm}$ in the case 
of metallic ferromagnets on a semiconductor substrate. It also makes it possible to measure the magnetic properties of ferromagnetic films with high accuracy.

Through detailed consideration of the problem, it becomes evident that there can be distinguished the following task: the development of magneto-ellipsometry technique which is based on the magneto-optical transverse Kerr effect and realized on a spectral ellipsometer with a static zero circuit with an external magnetic field applied to the sample. The solution is the use of models of ellipsometry of layered systems, developed for the case of joint analysis of the results of the reflective spectral ellipsometry measurements and changes in the ellipsometric parameters of a ferromagnetic sample appeared due to its magnetization reversal. The scale of this problem is such that the developed technique for modeling a multilayer ferromagnetic medium can be applied to any ferromagnetic, semiconductor and dielectric materials for analysis on any ellipsometric equipment.

The novelty is based on the fact that magneto-ellipsometry, as a method of studying ferromagnetic nanostructures, is developed rather slowly because of the complexity in the experimental data processing and their interpretation [1]. Despite such obvious advantages as high surface sensitivity, high speed and absence of influence on the sample, the method is less popular than microscopy and magnetometry because of the need to solve the inverse ellipsometry problem [2]. These difficulties repel many researchers from the use of ellipsometric techniques. Furthermore, if we consider the special case of optical and magneto-optical studies in the worldwide practice, mostly ex situ studies are carried out in order to measure the off-diagonal components of the permittivity tensor. Investigations of the samples properties are performed outside the process chamber, where they are synthesized [3-6]. Attempts to obtain information on the diagonal and offdiagonal components of the permittivity tensor simultaneously are few, for example, they were undertaken in [7], but the authors had to use magnetometry to measure the sample magnetization.

Therefore, it becomes obvious that the idea of developing an approach to solving an inverse problem of magneto-ellipsometry data analysis inside the vacuum technological chamber is of high interest and worthwhile to be considered.

\section{Section 1 Magneto-optical ellipsometry data processing}

This paper deals with developing the method so that it could be used for the case of multilayered ferromagnetic nanostructures study, improving its accessibility and understanding for the research community.

Successful obtaining the planned results was assumed due to the already existing developments in magneto-ellipsometry and methods of formation and investigation of ferromagnetic films made of pure iron and its silicides. At the moment, a number of works have been carried out in order to develop a set-up for thin-film structures synthesis and their in situ analysis using various models of reflective ferromagnetic nanostructures. Algorithms for solving the inverse magneto-ellipsometry problem for isotropic reflective layered systems with a ferromagnetic layer, described by a model of the homogeneous semi-infinite medium, a single-layer model and a two-layered model, are developed and implemented [8-10]. So this study logically continues our previous research in the field of layered nanostructures investigation by means of magneto-ellipsometry technique. Now it is time to generalise the approach for the case of a multilayered structure with ferromagnetic layer. Still we are interested in a structure with one ferromagnetic layer (FM) and others being nonferromagnetic (non-FM). We assume that the structures under investigation are isotropic.

The general idea of the approach to the inverse problem is already published [11]. Here for the convenience of the reader we provide a brief description of it as the following algorithm is based on the previous research. In short, we analyse the basic equation of ellipsometry [12]

$$
\rho=\operatorname{tg} \psi \exp (i \Delta)=\frac{R_{p}}{R_{S}}
$$


and Maxwell's equations in geometry of transverse magneto-optical Kerr effect in order to find out all elements of the dielectric permittivity tensor which in this case looks like

$$
[\varepsilon]=\left[\begin{array}{ccc}
\varepsilon_{11} & \varepsilon_{12} & 0 \\
\varepsilon_{21} & \varepsilon_{22} & 0 \\
0 & 0 & \varepsilon_{33}
\end{array}\right]=\left[\begin{array}{ccc}
\varepsilon_{11}^{\prime}-i \varepsilon_{11}^{\prime \prime} & -i\left(\varepsilon_{12}^{\prime}-i \varepsilon_{12}^{\prime \prime}\right)\left(Q_{1}-i Q_{2}\right) & 0 \\
i\left(\varepsilon_{12}^{\prime}-i \varepsilon_{12}^{\prime \prime}\right)\left(Q_{1}-i Q_{2}\right) & \varepsilon_{11}^{\prime}-i \varepsilon_{11}^{\prime \prime} & 0 \\
0 & 0 & \varepsilon_{11}^{\prime}-i \varepsilon_{11}^{\prime \prime}
\end{array}\right] .
$$

It means that we can obtain optical parameters (refractive index $n$ and extinction coefficient $k$ ) and complex magneto-optical Voight parameter $\left(Q=Q_{1}-i Q_{2}\right)$ from the measurements by means of spectral ellipsometry (measured ellipsometric parameters $\left.\psi_{0}, \Delta_{0}\right)$ and magneto-ellipsometry $(\delta \psi$, $\delta \Delta)$. The idea is that in order to extract the interested parameters we need to analyse reflection coefficients $(R p, R s)$ of the structure and to find out separately the values of magnetic and nonmagnetic real and imaginary parts of $R p, R s$ in the basic equation (1), so we consider them as:

$$
\begin{aligned}
& R_{p}=R_{p 0}+R_{p 1}=R_{p 0}^{\prime}+R_{p 1}^{\prime}-i\left(R_{p 0}^{\prime \prime}+R_{p 1}^{\prime \prime}\right) \\
& R_{S}=R_{S 0}=R_{S 0}^{\prime}-i R_{S 0}^{\prime \prime}
\end{aligned}
$$

where subindices 0 and 1 correspond to non-magnetic and magnetic summands, ' and " - to real and imaginary parts, $\mathrm{p}$ and $\mathrm{s}$ state for $\mathrm{p}$ - and s-polarization of the incident light, respectively. After a number of mathematical actions we achieve the following expressions:

$$
\begin{aligned}
& \delta \Delta=\Delta-\Delta_{0}=\operatorname{acrtg} \frac{R_{S 0}^{\prime \prime}\left(R_{p 0}^{\prime}+R_{p 1}^{\prime}\right)-R_{S 0}^{\prime}\left(R_{p 0}^{\prime \prime}+R_{p 1}^{\prime \prime}\right)}{R_{S 0}^{\prime}\left(R_{p 0}^{\prime}+R_{p 1}^{\prime}\right)+R_{S 0}^{\prime \prime}\left(R_{p 0}^{\prime \prime}+R_{p 1}^{\prime \prime}\right)}-\Delta_{0}, \\
& \delta \psi=\psi-\psi_{0}=\operatorname{arctg}\left(\sqrt{1+\frac{\left(R_{p 1}^{\prime 2}+R_{p 1}^{\prime \prime 2}+2\left(R_{p 0}^{\prime} R_{p 1}^{\prime}+R_{p 0}^{\prime \prime} R_{p 1}^{\prime \prime}\right)\right)\left(R_{S 0}^{\prime 2}+R_{S 0}^{\prime \prime 2}\right)}{\left(R_{p 0}^{\prime} R_{S 0}^{\prime}+R_{p 0}^{\prime \prime} R_{S 0}^{\prime \prime}\right)^{2}+\left(R_{S 0}^{\prime \prime} R_{p 0}^{\prime}-R_{p 0}^{\prime \prime} R_{S 0}^{\prime}\right)^{2}}} \operatorname{tg}\left(\psi_{0}\right)\right)-\psi_{0},
\end{aligned}
$$

where $\psi_{0}$ and $\Delta_{0}$ are ellipsometry parameters measured without magnetic field application, $\delta \psi$ and $\delta \Delta$ are the values of ellipsometry parameters changes during magnetization reversal. More details about the general approach can be found at [11], about finding optical properties of a layer - [13].

As for the algorithm for data analysis from multilayered structures which is presented in this paper (Fig. 1), it is realized in MATLAB as this environment provides opportunity to deal with big arrays and matrices directly [14] and to use several Matlab functions which are called by the main program. In the main program the researcher has an opportunity to set himself the following values: a number of layers in a structure (the substrate is not supposed to be numbered) $i$, the number of the ferromagnetic layer in the structure $i_{F M}$, an incidence angle $\varphi_{0}$, optical parameters $n_{i}, k_{i}$ of substrate and other layers (except parameters of the ferromagnetic as they are a subject to be investigated), arrays of experimental data such as magneto-optical ellipsometry $(\delta \psi, \delta \Delta)$, spectral ellipsometry $\left(\psi_{0}\right.$, $\Delta_{0}$ ), thicknesses of layers (they should be defined from ellipsometry data in advance) $d_{i}$. All these parameters help to generate $l x \times i$ matrix of optical thickness of the layer $\beta_{i}, l x \times(i+1)$ matrix of incidence angle cosine and refraction angles cosine, $l x \times(i+1)$ matrix of incidence angle sine and refraction angles sine, $l x \times(i+1)$ matrix of complex refraction index of the layer $N_{i}$, where $l x$ and $i$ in matrices dimensions are equal to number of wavelengths in spectrum and number of layers in a structure, respectively.

As soon as all necessary values are set, the main program calls other programs which make intermediate calculations of matrices and coefficients reported below. There are to be calculated layer matrices $L_{i}$ and interface matrices $I_{a b}[12]$ 


$$
\begin{aligned}
& \hat{L}_{i}=\left[\begin{array}{cc}
\exp \left(j \beta_{i}\right) & 0 \\
0 & \exp \left(-j \beta_{i}\right)
\end{array}\right], \\
& \hat{I}_{a b}=\left[\begin{array}{cc}
\frac{1}{t_{a b}} & -\frac{r_{b a}}{t_{a b}} \\
\frac{r_{a b}}{t_{a b}} & \frac{t_{b a} t_{a b}-r_{a b} r_{b a}}{t_{a b}}
\end{array}\right]=\frac{1}{t_{a b}}\left[\begin{array}{cc}
1 & -r_{b a} \\
r_{a b} & t_{b a} t_{a b}-r_{a b} r_{b a}
\end{array}\right],
\end{aligned}
$$

where

$$
\beta_{i}=\frac{2 \pi}{\lambda} N_{i} \cos \varphi_{i} d_{i}
$$

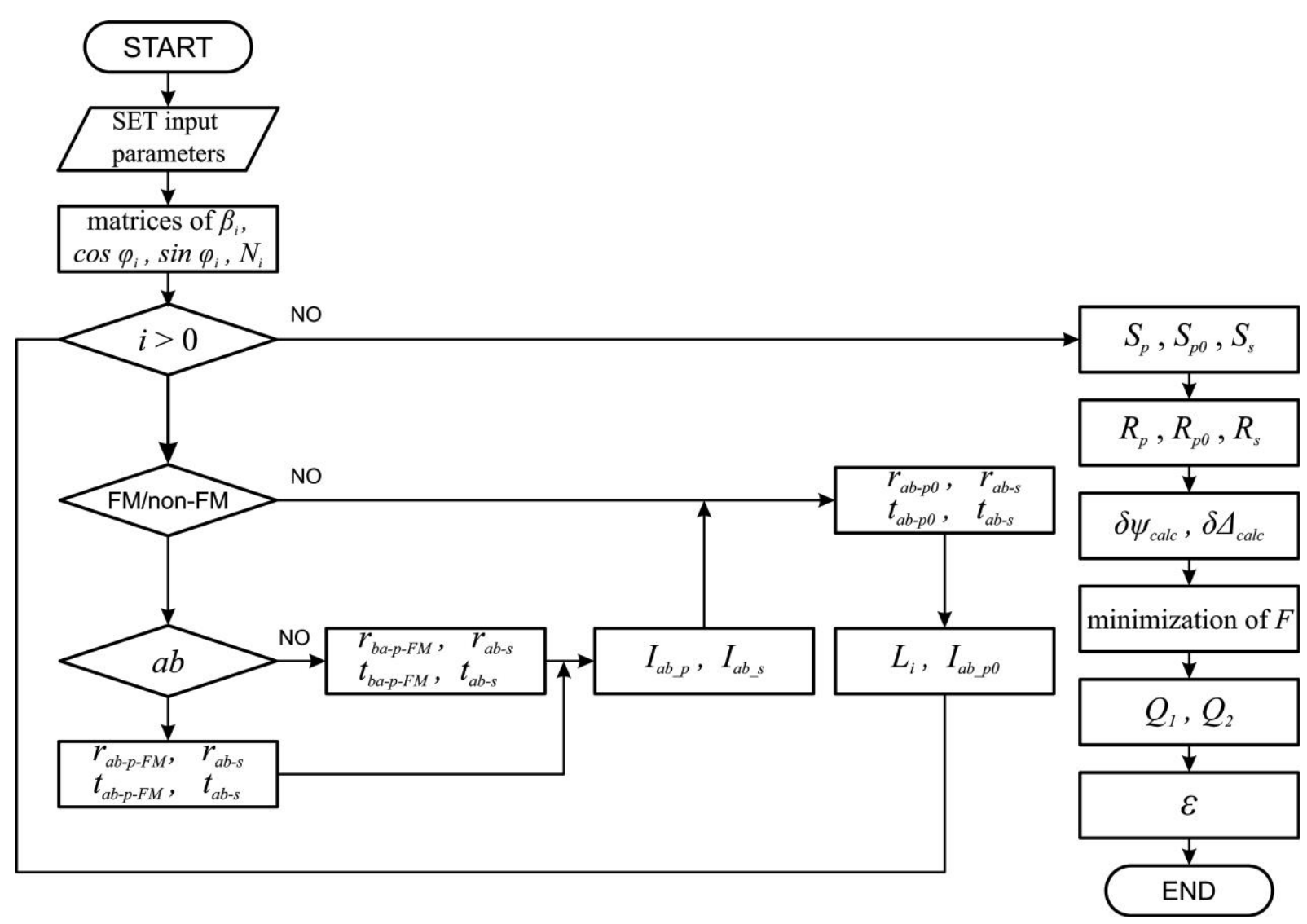

Figure 1. The flowchart of the algorithm for data analysis from multilayered structures

There is one layer matrix per each layer and three interface matrices for each interface. The reason is that layer matrices do not depend on the polarization but we have to calculate two interface matrices for $p$-polarisation of the incident light (they are marked as $p_{0}$ - for non-magnetic case, $p-$ for magnetic case) and one interface matrix corresponding to $s$-polarization of the incident light as it is not effected by the magnetic field at all. These matrices involve four transmission coefficients $(t)$ and four reflection coefficients $(r)$ use which depend in their turn on whether the interface is between FM/non-FM (subindex $F M$ ) or non-FM/non-FM layers (without subindex $F M$ ). Also it is essential to take into account the direction of the light beam in a film: subindex $a b$ corresponds to the light propagation from the film surface towards the substrate, while subindex $b a$ corresponds to the backward light direction (see Table 1): 


$$
\begin{aligned}
& t_{a b_{-} p_{-} F M}=\frac{2 N_{a} \cos \varphi_{a}}{N_{b} \cos \varphi_{a}+N_{a} \cos \varphi_{b}}+\frac{2 Q N_{a}^{3} \sin \varphi_{a} \cos \varphi_{a}}{N_{b}\left(N_{b} \cos \varphi_{a}+N_{a} \cos \varphi_{b}\right)^{2}}, \\
& t_{b a_{-} p_{-} F M}=\frac{2 N_{b} \cos \varphi_{b}}{N_{b} \cos \varphi_{a}+N_{a} \cos \varphi_{b}}-i \frac{2 Q N_{b}^{3} \sin \varphi_{b} \cos \varphi_{b}}{N_{a}\left(N_{b} \cos \varphi_{a}+N_{a} \cos \varphi_{b}\right)^{2}}, \\
& t_{a b_{-} p 0}=\frac{2 N_{a} \cos \varphi_{a}}{N_{b} \cos \varphi_{a}+N_{a} \cos \varphi_{b}}, \\
& t_{a b_{-} S}=\frac{2 N_{a} \cos \varphi_{a}}{N_{a} \cos \varphi_{a}+N_{b} \cos \varphi_{b}}, \\
& r_{a b_{-} p_{-} F M}=\frac{N_{b} \cos \varphi_{a}-N_{a} \cos \varphi_{b}}{N_{b} \cos \varphi_{a}+N_{a} \cos \varphi_{b}}-i \frac{2 Q N_{a}^{2} \sin \varphi_{a} \cos \varphi_{a}}{\left(N_{b} \cos \varphi_{a}+N_{a} \cos \varphi_{b}\right)^{2}}, \\
& r_{a b_{-} p 0}=\frac{N_{b} \cos \varphi_{a}-N_{a} \cos \varphi_{b}}{N_{b} \cos \varphi_{a}+N_{a} \cos \varphi_{b}}, \\
& r_{b a_{-} p_{-} F M}=\frac{N_{a} \cos \varphi_{b}-N_{b} \cos \varphi_{a}}{N_{a} \cos \varphi_{b}+N_{b} \cos \varphi_{a}}+i \frac{2 Q N_{b}^{2} \sin \varphi_{b} \cos \varphi_{b}}{\left(N_{a} \cos \varphi_{b}+N_{b} \cos \varphi_{a}\right)^{2}}, \\
& r_{a b_{-} S}=\frac{N_{a} \cos \varphi_{a}-N_{b} \cos \varphi_{b}}{N_{a} \cos \varphi_{a}+N_{b} \cos \varphi_{b}} .
\end{aligned}
$$

Table 1 Correspondence of transmission and reflection coefficients to the interface type

\begin{tabular}{|l|l|l|}
\hline Interface type & Transmission coefficients & Reflection coefficients \\
\hline FM/non-FM, $a b$ direction & $t_{a b-p-F M}, t_{a b-s}$ & $r_{a b-p-F M,}, r_{a b-s}$ \\
\hline FM/non-FM, $b a$ direction & $t_{b a-p-F M}, t_{a b-s}$ & $r_{b a-p-F M,} r_{a b-s}$ \\
\hline non-FM/non-FM, $a b$ direction & $t_{a b-p 0, \quad t_{a b-s}}$ & $r_{a b-p 0}, r_{a b-s}$ \\
\hline non-FM/non-FM, $b a$ direction [12] & $\left(1-r_{a b-p 0}^{2}\right) / t_{a b-p 0, \quad\left(1-r_{a b-s}^{2}\right) / t_{a b-s}}$ & $-r_{a b-p 0,}, r_{a b-s}$ \\
\hline
\end{tabular}

As there are three interface matrices per each interface, the next step is calculation of three scattering S-matrices which correspond to the whole layered nanostructure in case of different light polarization and magnetic field presence $\left(S_{p}, S_{p 0}\right.$ and $\left.S_{s}\right)$. In general, the formula is

$$
\hat{S}=\hat{I}_{01} \hat{L}_{1} \hat{I}_{12} \hat{L}_{2} \ldots \hat{I}_{(j-1) j} \hat{L}_{j} \ldots \hat{L}_{m} \hat{I}_{m(m+1)}
$$

To achieve $S_{p 0}$ matrix it is necessary to use only corresponding $p 0$ interface matrices in (18), to achieve $S_{s}$ matrix - to use only corresponding $s$ interface matrices, while for matrix $S_{p}$ it is essential to use two $p$ and others being $p 0$ interface matrices as only one layer is ferromagnetic in the nanostructure which leads to two interfaces characterized by $p$ interface matrices.

Then S-matrices elements $(2,1)$ and $(1,1)$ are used to calculate real and imaginary parts of full reflection coefficients of the structure $R_{p}, R_{p 0}, R_{s}[12]$ :

$$
\begin{aligned}
& R_{p}=R_{p 0}+R_{p 1}=\frac{S_{21 p}}{S_{11 p}}, \\
& R_{p 0}=\frac{S_{21 p 0}}{S_{11 p 0}}, \\
& R_{S}=\frac{S_{21 S}}{S_{11 S}} .
\end{aligned}
$$


After that $\delta \psi$ and $\delta \Delta$ are calculated by using expressions (5-6). The last step is using the NelderMead method [15] for minimization of the function of the sum of squares of differences of experimental and calculated $\delta \psi$ and $\delta \Delta \quad F=\left\{\left(\delta \psi_{\text {exp }}-\delta \psi_{\text {calc }}\right)^{2}+\left(\delta \Delta_{\text {exp }}-\delta \Delta_{\text {calc }}\right)^{2}\right\}$, which finally provides us with the best values of real and imaginary parts of Voight magneto-optical parameter $Q$. It means that by using (2) all non-diagonal elements of dielectric permittivity tensor can be found out.

\section{Summary}

In conclusion, we offer the method of magneto-ellipsometry data analysis which has a number of advantages: 1) it can be used both $\boldsymbol{e x}$ situ and in situ with no changes needed to be made according to the choice, 2) there is no need to use Muller matrix formalism which means no need to carry out additional measurements, 3 ) it is applicable for using inside vacuum chamber and controlling the nanostructures growth, 4) as a result spectra of dielectric permittivity tensor can be obtained. At this moment it can be used for analyzing structures described by a model of the homogeneous semiinfinite medium, a single-layer model, a two-layered model and a multilayered model with one ferromagnetic layer. In future multilayered structures with several FM layers will be studied.

\section{Acknowledgments}

The work was funded by state budget project No. 0356-2017-0030 "Condensed state physics: dielectrics, magnets, multiferroics, superconductors. Synthesis, study of physical properties, theory"

\section{References}

[1] G. Neuber, et al., Temperature-dependent spectral generalized magneto-optical ellipsometry, Appl. Phys. Lett. 83 (2003) 4509.

[2] H. Fujiwara, Spectroscopic ellipsometry principles and applications, John Wiley \& Sons, Chichester, 2007.

[3] Q.J. Nederpel, J.W.D. Martens, Magneto-optical ellipsometer, Rev. Sci. Instrum. 56 (1985) 687. [4] A. Berger, M.R. Pufall, Generalized magneto-optical ellipsometry, Appl. Phys. Lett. 71 (1997) 965.

[5] K. Mok et al., Vector-magneto-optical generalized ellipsometry, Rev. Sci. Instrum. 82 (2011) 33112.

[6] K. Mok et al., Thickness independent magneto-optical coupling constant of nickel films in the visible spectral range, J. Appl. Phys. 110 (2011) 123110.

[7] K. Mok et al., Magneto-optical coupling in ferromagnetic thin films investigated by vectormagneto-optical generalized ellipsometry, Phys. Rev. 84 (2011) 094413.

[8] O.A. Maximova, et al., In situ magneto-optical ellipsometry data analysis for films growth control, JMMM 440 (2017) 196-198.

[9] O.A. Maximova, et al., Single-layer model of reflective nanostructures for magneto-ellipsometry data analysis, IOP Conference Series: Materials Science and Engineering 155 N. 1 (2016) 012030.

[10] O.A. Maximova, et al., Two-layer model of reflective ferromagnetic films in terms of magnetooptical ellipsometry studies, SibFU Journal. Mathematics \& Physics 10 N.2 (2017) 223-232.

[11] O.A. Maximova, et al., Features of the ellipsometric investigation of magnetic nanostructures, J. Struct. Chem. 55 (6) (2014) 1134-1141.

[12] R.M.A. Azzam, N.M. Bashara, Ellipsometry and polarized light, North-Holland Pub. Co, Amsterdam, 1977.

[13] I.A. Tarasov, et al., Quick ellipsometric technique for determining the thicknesses and optical constant profiles of $\mathrm{Fe} / \mathrm{SiO} 2 / \mathrm{Si}(100)$ nanostructures during growth, Tech. Phys. 57 (2012) 1225.

[14] Information on https://www.mathworks.com/products/matlab.html matlab

[15] J.A. Nelder, A.D. Mead, A simplex method for function minimization, Comput. J. 7 (1965)

$308-313$. 